\title{
NILPOTENT INVERSE SEMIGROUPS WITH CENTRAL IDEMPOTENTS
}

BY

\author{
G. KOWOL AND H. MITSCH
}

\begin{abstract}
An inverse semigroup $S$ with central idempotents, i.e. a strong semilattice of groups, will be called nilpotent, if it is finite and if for each prime divisor $p_{i}$ of the orders of the structure groups of $S$ the sets $P_{i}=\left\{s \in S \mid o(s)=p_{i}^{k_{\mathrm{v}}}, k_{s} \geqslant 0\right\}$ are subsemigroups of $S$. If $S$ is a group, then $P_{i}$ are exactly the Sylow $p_{i}$-subgroups of the group. A theory similar to that given by $\mathrm{W}$. Burnside for finite nilpotent groups is developed introducing the concepts of ascending resp. descending central series in an inverse semigroup, and it is shown that almost all of the well-known properties of finite nilpotent groups do hold also for the class of finite inverse semigroups with central idempotents.
\end{abstract}

1. Introduction. In the theory of finite groups the concept of nilpotency plays a central role. There are a number of equivalent possibilities to define a nilpotent group-for example see Huppert [5, III.2.3]:

(a) For every prime divisor $p$ of the order of $G$ there exists one and only one Sylow $p$-subgroup of $G$.

(b) $G$ is the direct product of its Sylow subgroups.

(c) The descending central series of $G$ terminates in the unity of $G$.

(d) The ascending central series of $G$ terminates in $G$.

(e) Every homomorphic image of $G$ different from the identity has a nontrivial center.

(f) Elements of $G$ having relatively prime orders commute.

In the following we try to generalise the notion of nilpotency in groups to the class of inverse semigroups $S$. It turns out that properties similar to (a)-(f) above do hold only under the additional assumption that the idempotents of $S$ are central. Thus we suppose $S$ to be a Clifford semigroup. Recall (see Howie [3] or Clifford-Preston [2]) that a Clifford semigroup is defined as a semigroup $(S, \cdot)$, such that

(1) for every $a \in S$ there exists exactly one $a^{-1} \in S$ with $a a^{-1} a=a, a^{-1} a a^{-1}=a^{-1}$,

(2) $a e=e a$ for every $a \in S, e \in E(S)$ (= the semilattice of idempotents in $S$ ).

Equivalent to conditions (1) and (2) is the statement that $S$ is a strong semilattice of groups $G_{\alpha}$, i.e. $S=\cup G_{\alpha}$, there is a congruence $\rho$ on $S$ such that $S / \rho=Y$ is a semilattice isomorphic to $E(S)$, every element of $S / \rho$ being a subgroup $G_{\alpha}$ of $S$ with identity $e_{\alpha}$, and to each pair $\alpha, \beta \in Y$ with $\alpha>\beta$ a homomorphism $\varphi_{\alpha, \beta}: G_{\alpha} \rightarrow G_{\beta}$ is defined satisfying $\varphi_{\beta, \gamma} \circ \varphi_{\alpha, \beta}=\varphi_{\alpha, \gamma}$ for all $\alpha>\beta>\gamma$; furthermore for $a_{\alpha} \in G_{\alpha}$,

Received by the editors August 13, 1980 and, in revised form, March 4, 1981.

1980 Mathematics Subject Classification. Primary 20M10. 
$b_{\beta} \in G_{\beta}$ we have $a_{\alpha} b_{\beta}=\varphi_{\alpha, \gamma}\left(a_{\alpha}\right) \varphi_{\beta, \gamma}\left(b_{\beta}\right) \in G_{\gamma}$, where $\gamma=\alpha \beta$ in the semilattice $Y$ (see Clifford [1]).

Since normal subgroups of groups have to be replaced by congruences considering semigroups, we derive some results on congruences of Clifford semigroups in $\$ 2$ using the notion of congruence pairs introduced by Petrich [8]. The concept of the commutator of two elements in a group can easily be generalised to inverse semigroups; we shall consider it in detail in $\$ 3$, since it will be extremely useful in the investigation of nilpotent Clifford semigroups. The definition of nilpotency will be given in $\$ 4$, where we shall develop a theory analogous to that established by Burnside for nilpotent groups.

2. Normal subsemigroups and congruence pairs. We recall the definition of congruence pairs and corresponding congruences given by Petrich [8], which yields the knowledge of all homomorphic images of an arbitrary inverse semigroup.

Let $S$ be an inverse semigroup with semilattice of idempotents $E(S)$; a subsemigroup $N$ of $S$ is called "normal", if (1) $a \in N \Rightarrow a^{-1} \in N$; (2) $E(S) \subseteq N$; (3) $s^{-1} N s \subseteq N$ for all $s \in S$. A congruence $\xi$ on $(E(S), \cdot)$ is called "normal", if $e \xi f$ $(e, f \in E(S))$ implies $s^{-1} e s \xi s^{-1} f s$ for every $s \in S$.

A pair $(\xi, \mathrm{e} N), \xi$ and $N$ normal, is called "congruence pair of $S$ ", if

(1) $a e \in N, e \xi a^{-1} a(a \in S, e \in E(S)) \Rightarrow a \in N$.

(2) $a \in N \Rightarrow a^{-1} e a \xi a^{-1} a e$ for every $e \in E(S)$.

If $(\xi, N)$ is a congruence pair of $S$, then $\kappa(\xi, N)$ defined by

$$
a \kappa(\xi, N) b \Leftrightarrow a^{-1} a \xi b^{-1} b \text { and } a b^{-1} \in N,
$$

is a congruence on $S$ whose restriction to $E(S), \operatorname{tr} \kappa$, equals $\xi$, and whose kernel ker $\kappa=\{s \in S \mid$ sкe for some $e \in E(S)\}$ is $N$. Conversely, if $\rho$ is any congruence on $S$, then $(\operatorname{tr} \rho, \operatorname{ker} \rho)$ is a congruence pair of $S$ and $\rho=\kappa(\operatorname{tr} \rho, \operatorname{ker} \rho)$; the correspondence $(\xi, N) \rightarrow \kappa(\xi, N)$ resp. $\rho \rightarrow(\operatorname{tr} \rho, \operatorname{ker} \rho)$ is one-to-one.

In the following only those congruence pairs $(\xi, N)$ will be needed where $\xi=\varepsilon$, the identity relation on $E(S)$, which is normal trivially. The corresponding congruences $\kappa(\varepsilon, N)$ clearly are idempotent-separating, i.e. $e \kappa f, e, f \in E(S) \Rightarrow e=f$. Concerning the existence of such pairs we have by Mitsch [7, Proposition 2]: If $S$ is an inverse semigroup and $N$ a normal subsemigroup of $S$, then $(\varepsilon, N)$ is a congruence pair of $S$, iff $a e=e a$ for every $a \in N, e \in E(S)$; in particular: every pair $(\varepsilon, N)$ is a congruence pair, iff $S$ is a Clifford semigroup.

LEMMA 2.1. Let $S$ be an inverse semigroup and $Z(S)=\{a \in S \mid$ as $=$ sa for every $s \in S\}$. Then $(\varepsilon, Z(S))$ is a congruence pair of $S$ iff $S$ is a Clifford semigroup.

Proof. In order to prove sufficiency, we have only to show that $(Z(S), \cdot)$ is a normal subsemigroup of $S$. By hypothesis $E(S) \subseteq Z(S)$; $a, b \in Z(S)$ implies $a b \in$ $Z(S)$ evidently. Let $a \in Z(S)$; then $a s^{-1}=s^{-1} a$ for every $s \in S$, thus $s a^{-1}=a^{-1} s$ by taking inverses and $a^{-1} \in Z(S)$. Finally let $a \in Z(S), x, s \in S$; then

$$
s\left(x^{-1} a x\right)=s\left(x^{-1} a\right) x=s a x^{-1} x=a s x^{-1} x=a\left(x^{-1} x\right) s=\left(x^{-1} a x\right) s,
$$

thus $x^{-1} a x \in Z(S)$ for every $x \in S$. Necessity is clear, since $E(S) \subseteq Z(S)$ means that the idempotents of $S$ are central. 
LEMMA 2.2. Let $S$ be an inverse semigroup. Then $S / \kappa(\varepsilon, S) \cong E(S)$ iff $S$ is a Clifford semigroup. If $S / \kappa(\varepsilon, N) \cong E(S)$ for some normal subsemigroup $N$ of $S$, then $N=S$.

Proof. The first assertion is Theorem 2.6 of Howie [4]. The second statement is clear, since $S / \kappa(\varepsilon, N) \cong E(S) \cong S / \kappa(\varepsilon, S)$ implies $\kappa(\varepsilon, N)=\kappa(\varepsilon, S)$, so that by Petrich [8, Corollary 4.5] follows $N=S$.

LEMMA 2.3. Let $S$ be an inverse semigroup. (a) If $\rho$ is any congruence on $S$ and $K$ is a normal subsemigroup of $S / \rho$, then $\varphi^{-1}(K)$ is normal in $S$ ( $\varphi$ the natural homomorphism of $\rho$ ). (b) If $\rho$ is any congruence of $S$ with kernel $N$, then $\varphi(N)=E(S / \rho)$.

Proof. (a) is evident. (b) Let $a \in N$; then $[a]_{\rho}=\left[a^{-1} a\right]_{\rho}$, since

$$
a^{-1} a \xi\left(a^{-1} a\right)^{-1}\left(a^{-1} a\right)=a^{-1} a \text { and } a\left(a^{-1} a\right)^{-1}=a a^{-1} a=a \in N
$$

imply $a \rho a^{-1} a$; consequently $[a]_{\rho} \in E(S / \rho)$ and $\varphi(a) \in E(S / \rho)$. Conversely let $[a]_{\rho} \in E(S / \rho)$; then by the lemma of Lallement $[6]^{[}[a]_{\rho}=[e]_{\rho}$ for some idempotent $e \in E(S) \subseteq N$, thus $\varphi(a)=\varphi(e) \in \varphi(N)$ and $[a]_{\rho} \in \varphi(N)$.

LEMMA 2.4. Let $S$ be a Clifford semigroup. (a) If $\rho$ is a congruence on $S$, then $S / \rho$ is a Clifford semigroup. (b) If $M, N$ are normal subsemigroups of $S$, then $N \cdot M$ is normal in $S$, too.

Proof. (a) First $S / \rho$ is an inverse semigroup (see Clifford-Preston [2]); let $[a]_{\rho} \in E(S / \rho)$, then $[a]_{\rho}=[e]_{\rho}$ for some $e \in E(S)$ by Lallement $[6]$ and $[a]_{\rho}[s]_{\rho}=$ $[e]_{\rho}[s]_{\rho}=[e s]_{\rho}=[s e]_{\rho}=[s]_{\rho}[a]_{\rho}$ for every $[s]_{\rho} \in S / \rho$, i.e. the idempotents of $S / \rho$ are central.

(b) Let $a, b \in N \cdot M$; then $a=x_{1} y_{1}, b=x_{2} y_{2}, x_{1}, x_{2} \in N, y_{1}, y_{2} \in M$; thus $a b=x_{1} y_{1} x_{2} y_{2}=x_{1} y_{1}\left(x_{2} x_{2}^{-1} x_{2}\right) y_{2}=x_{1} y_{1}\left(x_{2} x_{2}^{-1}\right) x_{2} y_{2}=x_{1} x_{2}\left(x_{2}^{-1} y_{1} x_{2}\right) y_{2}=$ $\left(x_{1} x_{2}\right) m y_{2}=n\left(m y_{2}\right)=n m_{1} \in N \cdot M$; if $a \in N \cdot M$, then $a=x y$ and $a^{-1}=y^{-1} x^{-1}$ $=y^{-1}\left(x^{-1} x x^{-1}\right)=\left(x^{-1} x\right)\left(y^{-1} x^{-1}\right) \in N \cdot M ; E(S) \subseteq N \cdot M$, since $e=e \cdot e \in N \cdot M$ for every $e \in E(S)$; finally let $a \in N \cdot M, s \in S$; then $a=x y$ and $s^{-1} a s=s^{-1} x y s=$ $s^{-1} x y\left(s s^{-1}\right) s=\left(s^{-1} x s\right)\left(s^{-1} y s\right) \in N \cdot M$.

3. Commutators in inverse semigroups. The concept of the "commutator of two elements $x, y$ of an inverse semigroup $S$ " can be defined in the same way as for a group: $[x, y]=x^{-1} y^{-1} x y$. Similarly by the "commutator semigroup $T^{\prime}$ generated by the subset $T$ of $S$ " we mean the least inverse subsemigroup of $S$ containing all the commutators $[x, y]$ of arbitrary elements $x, y$ of $T$. For $T=S$ we call $S^{\prime}$ the "commutator semigroup of $S$ ".

Proposition 3.1. Let $S$ be a Clifford semigroup. Then the following hold:

(a) Two elements $a, b \in S$ commute iff $[a, b] \in E(S)$.

(b) $(S, \cdot)$ is commutative iff $S^{\prime}=E(S)$.

(c) If $K, N$ are two normal subsemigroups of $S$ such that $K \cap N=E(S)$, then every pair of elements $x \in K, y \in N$ commute, i.e. $x y=y x$. 
Proof. (a) Let $a b=b a$ for two elements $a, b$ of $S$; then $[a, b]=a^{-1} b^{-1} a b=$ $a^{-1}\left(b^{-1} b\right) a=\left(a^{-1} a\right)\left(b^{-1} b\right) \in E(S)$. Conversely let $[a, b] \in E(S)$ for some $a, b \in S$; then

$$
b a[a, b]=b\left(a a^{-1}\right) b^{-1} a b=\left(b b^{-1}\right)\left(a a^{-1} a\right) b=\left(b b^{-1}\right) a b=a b b^{-1} b=a b ;
$$

this means in the natural order of $S$, that $b a \geqslant a b$; but $[a, b] \in E(S) \Rightarrow[a, b]=$ $[a, b]^{-1}=[b, a]$, thus $a b[a, b]=a b[b, a]=b a$, and $a b \geqslant b a$.

(b) If $(S, \cdot)$ is commutative, then by (a) just proved: $[a, b] \in E(S)$ for all $a, b \in S$, which means that $E(S)$ being an inverse subsemigroup of $S$ contains all the commutators of $S$. By the minimality of the commutator semigroup $S^{\prime}$ we get $S^{\prime} \subseteq E(S)$; on the other hand we have for every $e \in E(S)$ that $e=[e, e] \in S^{\prime}$ and equality follows. The converse statement is clear by (a).

(c) Let $a \in K, b \in N$ be arbitrary elements; then $[a, b]=a^{-1}\left(b^{-1} a b\right)=k_{1} k_{2} \in K$, since $K$ is normal; analogously $[a, b]=\left(a^{-1} b^{-1} a\right) b \in N$. Thus we get $[a, b] \in K \cap$ $N=E(S)$ and we conclude by (a), that $a b=b a$.

The condition that $S$ be a Clifford semigroup in Proposition 3.1 cannot be weakened, as the following corollary shows.

COROllaRy 3.2. In an inverse semigroup $S[a, b] \in E(S)$ implies $a b=b a$ for elements $a, b$ of $S$ iff $S$ is a Clifford semigroup.

Proof. Sufficiency is obvious by Proposition 3.1(a).

Necessity. Let $a \in S, e \in E(S)$; then

$$
\left(a^{-1} e a\right)\left(a^{-1} e a\right)=a^{-1} e\left(a a^{-1}\right) e a=\left(a^{-1} a a^{-1}\right) e a=a^{-1} e a,
$$

thus $a^{-1} e a \in E(S)$ and therefore $[e, a]=e\left(a^{-1} e a\right) \in E(S)$. By hypothesis this implies $a e=e a$ for every $a \in S, e \in E(S)$, which means that the idempotents of $S$ are central.

The commutator semigroup $S^{\prime}$ of a Clifford semigroup $S$ is always a normal subsemigroup of $S$ : by definition $S^{\prime}$ is an inverse subsemigroup of $S ; E(S) \subseteq S^{\prime}$ trivially; let $a \in S^{\prime}, s \in S$, then $a$ is a product of commutators of $S$-suppose first that $a=[x, y], x, y \in S$; then

$$
\begin{aligned}
s^{-1} a s & =s^{-1}[x, y] s=s^{-1} x^{-1} y^{-1} x y s=s^{-1} x^{-1} y^{-1} x y\left(s s^{-1}\right) s \\
& =s^{-1} x^{-1}\left(s s^{-1}\right) y^{-1} x y s=\left(s^{-1} x^{-1} s\right)\left(s^{-1} y^{-1} s\right) s^{-1} x y s \\
& =\left(s^{-1} x^{-1} s\right)\left(s^{-1} y^{-1} s\right) s^{-1} x\left(s s^{-1}\right) y s=\left[s^{-1} x s, s^{-1} y s\right] \in S^{\prime} ;
\end{aligned}
$$

by a similar argument the general case can be shown, too. Now by Mitsch [7, Proposition 2], $(\varepsilon, N)$ is a congruence pair for any normal subsemigroup $N$ of an arbitrary Clifford semigroup $S$; thus $\kappa=\kappa\left(\varepsilon, S^{\prime}\right)$ is a congruence on $S$. For sake of convenience we shall write for the factor semigroup of $S$ with respect to the congruence $\kappa=\kappa\left(\varepsilon, S^{\prime}\right)$ in short: $S / S^{\prime}$; generally,

$S / N \ldots$ factor semigroup of $S$ with respect to the congruence $\kappa=\kappa(\varepsilon, N)$.

PROPOSITION 3.3. For any Clifford semigroup the following hold:

(a) $S / S^{\prime}$ is commutative.

(b) $S / \rho$ is commutative iff $\rho \geqslant \kappa=\kappa\left(\varepsilon, S^{\prime}\right)$.

(c) $S / N$ is commutative iff $N \supseteq S^{\prime}$. 
Proof. (a) Let $\kappa=\kappa\left(\varepsilon, S^{\prime}\right)$ and $[a]_{\kappa},[b]_{\kappa} \in S / S^{\prime}$ arbitrary; then

$$
(a b)^{-1} a b=b^{-1}\left(a^{-1} a\right) b=\left(a^{-1} a\right)\left(b^{-1} b\right)=a^{-1} b^{-1} b a=(b a)^{-1} b a
$$

and furthermore $(a b)(b a)^{-1}=a b a^{-1} b^{-1}=\left[a^{-1}, b^{-1}\right] \in S^{\prime}$; by definition of $\kappa\left(\varepsilon, S^{\prime}\right)$ this means that $a b \kappa\left(\varepsilon, S^{\prime}\right) b a$; consequently $[a]_{\kappa}[b]_{\kappa}=[a b]_{\kappa}=[b a]_{\kappa}=[b]_{\kappa}[a]_{\kappa}$ and $S / S^{\prime}$ is commutative.

(b) If $\rho \geqslant \kappa\left(\varepsilon, S^{\prime}\right)$, then by the second (Howie [3, Theorem I.5.6]) we have: $S / \rho \cong(S / \kappa) /(\rho / \kappa)$, where $[a]_{\kappa}(\rho / \kappa)[b]_{\kappa} \Leftrightarrow a \rho b$. By (a) the semigroup $\left(S / S^{\prime}, \cdot\right)$ is commutative, thus every factor semigroup of it, too, and so is $S / \rho$. Conversely let $S / \rho$ be commutative for some congruence $\rho$ on $S$. The congruence pair defining $\rho$ is given by $(\operatorname{tr} \rho, \operatorname{ker} \rho)$; comparing this with the congruence pair $\left(\varepsilon, S^{\prime}\right)$ in the ordering of Corollary 4.5 in Petrich [8] we have: $\varepsilon \leqslant \operatorname{tr} \rho$ trivially; moreover $S^{\prime} \subseteq \operatorname{ker} \rho$, since for any $x \in S^{\prime}, x=[s, t]$ it follows st $\rho t s$ ( $S / \rho$ is commutative), thus $t^{-1} s t \rho t^{-1} t s \rho s t^{-1} t$ and $s^{-1} t^{-1} s t \rho\left(s^{-1} s\right)\left(t^{-1} t\right)$, consequently $[s, t] \rho e$ for some $e \in E(S)$, which implies that $x=[s, t] \in \operatorname{ker} \rho$; also every product of commutators on $S$ belongs to $\operatorname{ker} \rho$ (since $\operatorname{ker} \rho$ is normal in $S$ ); hence we obtain $\left(\varepsilon, S^{\prime}\right) \leqslant(\operatorname{tr} \rho, \operatorname{ker} \rho)$, i.e. $\kappa=\kappa\left(\varepsilon, S^{\prime}\right) \leqslant$ $\rho=\rho(\operatorname{tr} \rho, \operatorname{ker} \rho)$.

(c) This is assertion (b) formulated by means of normal subsemigroups, since for any (normal) congruence $\xi$ on $E(S)$ we have $\xi \geqslant \varepsilon$ trivially.

4. Nilpotent Clifford semigroups. The 'best' definition of nilpotency for our purposes is that which corresponds to property (a) of finite nilpotent groups above: for every prime divisor of the order of the group there exists exactly one Sylow subgroup.

Definition. Let $S$ be a finite Clifford semigroup, $S=\cup G_{\alpha}, \alpha \in Y$. For every $s \in S$ let $o(s)$ denote the order of $s$ in the group $G_{\alpha}$, to which it belongs; further let $p_{1}, \ldots, p_{n}$ be all the prime divisors of the orders of the groups $G_{\alpha}, \alpha \in Y$. Then $S$ will be called "nilpotent", if the following subsets of $S$ are subsemigroups $(i=1, \ldots, n)$ :

$$
P_{i}=\left\{s \in S \mid o(s)=p_{i}^{k_{1}}, k_{s} \geqslant 0\right\} \text {. }
$$

Remarks. (1) If $S$ is a group of finite order, then $P_{i}$ are exactly the Sylow $p_{i}$-subgroups of $G$. Thus if $S$ is Clifford, we shall call $P_{i}$ the "Sylow $p_{i}$-subsemigroups of $S$ "-by definition, there exists one and only one such for every prime-divisor $p_{i}$ $(i=1, \ldots, n)$.

(2) $P_{i} \neq \varnothing(i=1, \ldots, n)$, since for every idempotent $e_{\alpha} \in E(S), \alpha \in Y$, and every prime $p_{i}(i=1, \ldots, n)$ we have: $o\left(e_{\alpha}\right)=1=p_{i}^{0}$ in $G_{\alpha}$ implying $E(S) \subseteq p_{i}$ for all $i$.

(3) Every subsemigroup $T$ of a nilpotent Clifford semigroup is nilpotent, too. In fact: since $S$ is finite, each of the defining groups $G_{\alpha}, \alpha \in Y$, is finite; thus for every $a \in T$, i.e. $a \in G_{\alpha}$ for some $\alpha \in Y$, the inverse $a^{-1}$ belongs to the subgroup of $G_{\alpha}$ generated by $a$ in $G_{\alpha}: a^{-1} \in\left\{a, a^{2}, \ldots, a^{r-1}\right\}$; this set belongs to $T$, since $T$ is a subsemigroup of $S$, consequently $(T, \cdot)$ is an inverse subsemigroup of $S$, which moreover has only central idempotents, trivially, i.e. $T$ is a Clifford semigroup. $T$ is nilpotent, since $A_{i}=\left\{x \in T \mid o(x)=p_{i}^{k}\right\}=P_{i} \cap T$, where $P_{i}$ and $T$ are both subsemigroups of $S$, consequently $A_{i}$ is a subsemigroup of $T$, too. 
Similarly to commutative Clifford semigroups (compare Corollary IV, 2.2 of Howie [3]) nilpotent Clifford semigroups can be described by their defining groups in a simple way - they are exactly those Clifford semigroups which are built up by nilpotent groups, providing enough examples of nilpotent Clifford semigroups.

Theorem 4.1. A finite Clifford semigroup $S$ is nilpotent iff each of the defining groups $G_{\alpha}, \alpha \in Y$, is nilpotent.

Proof. First let $G_{\alpha}$ be nilpotent for each $\alpha \in Y$; then every $G_{\alpha}$ has exactly one Sylow $p_{i}$-subgroup with $p_{i}$ prime divisor of the order of $G_{\alpha}$. Let $a, b \in P_{i}=\{s \in$ $\left.S \mid o(s)=p_{1}^{k_{1}}, k_{s} \geqslant 0\right\}$; then for $a \in G_{\alpha}, b \in G_{\beta}$ the elements $a$ resp. $b$ belong to the Sylow $p_{i}$-subgroup of $G_{\alpha}$ resp. $G_{\beta}$. If $\gamma=\alpha \beta$ in the semilattice $Y$, then $a b=$ $\varphi_{\alpha, \gamma}(a) \varphi_{\beta, \gamma}(b)$, where $\varphi_{\alpha, \gamma}: G_{\alpha} \rightarrow G_{\gamma}, \varphi_{\beta, \gamma}: G_{\beta} \rightarrow G_{\gamma}$ are the defining structure homomorphisms. Since any homomorphic image of a $p$-group is a $p$-group again, the elements $\varphi_{\alpha, \gamma}(a)$ resp. $\varphi_{\beta, \gamma}(b)$ belong to the Sylow $p_{i}$-subgroup of $G_{\gamma}$, consequently their product, too. Thus $o(a b)=p_{i}^{r}$ and $a b \in P_{i}$.

Conversely let $S$ be nilpotent and let $p_{i}(i=1, \ldots, k)$ be the prime divisors of the order of $G_{\alpha}, \alpha \in Y$ fixed. We show that $T_{i}=\left\{g \in G_{\alpha} \mid o(g)=p_{i}^{r}, r \geqslant 0\right\}, i=$ $1, \ldots, k$, is a subgroup of $G_{\alpha}$ : first we note that $T_{i}=G_{\alpha} \cap P_{i}$, where both $G_{\alpha}$ and $P_{i}$ are subsemigroups of $S$, thus $T_{i}$ also in $G_{\alpha}$; but since $G_{\alpha}$ is finite, $T_{i}$ is a subgroup of $G_{\alpha}$; now by definition $T_{i}$ is the only Sylow $p_{i}$-subgroup of $G_{\alpha}$ and we conclude by Huppert [5, Theorem III, 2.3], that $G_{\alpha}$ is nilpotent.

The property of $P_{i}$ being a subsemigroup in any nilpotent Clifford semigroup has several important consequences.

LEMMA 4.2. For any finite nilpotent Clifford semigroup the following hold:

(1) Every subsemigroup $P_{i}(i=1, \ldots, n)$ is normal in $S$.

(2) $\left(\prod_{i<k} P_{i}\right) \cap P_{k}=E(S)$ for every $k=2, \ldots, n$.

(3) $S=\prod_{i=1}^{n} P_{i}$, i.e. $s \in S \Rightarrow s=q_{1} \cdots q_{n}$ with $q_{i} \in P_{i}$.

(4) $P_{i} \cap P_{j}=E(S)$ for $i \neq j(i, j=1, \ldots, n)$.

Proof. (1) $\left(P_{i}, \cdot\right)$ is an inverse subsemigroup, since $x \in P_{i}$ implies $x \in G_{\alpha}$ for some $\alpha \in Y$ with $o(x)=p_{i}^{r}(r \geqslant 0)$; thus $x^{-1} \in G_{\alpha}$ with $o\left(x^{-1}\right)=o(x)$ and $x^{-1} \in P_{i}$. Because of $o(e)=p_{i}^{0}$ for every $e \in E(S)$ and every $i=1, \ldots, n$ we have $E(S) \subseteq P_{i}$. Finally let $a \in P_{i}, s \in S$; then $a \in G_{\alpha}, s^{-1} a s \in G_{\beta}$ for some $\alpha, \beta \in Y$; now $\left(s^{-1} a s\right)^{o(a)}=s^{-1} a\left(s s^{-1}\right) a\left(s s^{-1}\right) \cdots\left(s s^{-1}\right) a s=s^{-1}\left(s s^{-1}\right) a^{o(a)} s=s^{-1} e_{\alpha} s \in E(S)$, which implies that $o\left(s^{-1} a s\right)$ divides $o(a)=p_{i}^{r}$, thus $o\left(s^{-1} a s\right)=p_{i}^{t}(t \leqslant r)$ and $s^{-1} a s$ $\in P_{i}$.

(2) For $k=2, a \in P_{1} \cap P_{2}$ implies $o(a)=p_{1}^{r}=p_{2}^{t}\left(p_{1} \neq p_{2}\right)$, consequently $r=t$ $=0$ and $o(a)=1$, i.e. $a \in E(S)$; the opposite inclusion is trivial, since $E(S) \subseteq P_{1}$, $P_{2}$ by (1) above. Now by way of induction let $a \in\left(\prod_{i<k} P_{i}\right) \cap P_{k}$, i.e. $a \in P_{k}$ and $a \in \Pi_{i<k} P_{i}$, where $\left(\prod_{i<k-1} P_{i}\right) \cap P_{k-1}=E(S)$. Then $a=\left(q_{1} \cdots q_{k-2}\right) q_{k-1}$ with $q_{j} \in P_{j}, j=1, \ldots, k-1$, thus $a=b c$, where $b \in \prod_{i<k-1} P_{i}, c \in P_{k-1}$. By Lemma 2.4(b) and (1) above $\Pi_{i<k-1} P_{i}$ and $P_{k-1}$ are normal subsemigroups of $S$, so that $b c=c b$ by Proposition 3.1(c); generally: $(b c)^{n}=b^{n} c^{n}$ for every $n \in \mathbf{N}$. Let $o\left(q_{k-1}\right)$ $=t$, then $a^{t}=\left(q_{1} \cdots q_{k-2}\right)^{t} q_{k-1}^{t}=\left(q_{1} \cdots q_{k-2}\right)^{t} e$ with $e \in E(S)$; let $o\left(q_{k-2}\right)=r$; 
then $a^{t r}=\left(a^{t}\right)^{r}=\left(q_{1} \cdots q_{k-3}\right)^{t r} q_{k-2}^{t r} e=\left(q_{1} \cdots q_{k-3}\right)^{t r} f e$ with $e, f \in E(S)$, and so on: $a^{s}=e_{1} \cdots e_{k-1} \in E(S)$ where $s=o\left(q_{1}\right) \cdots o\left(q_{k-1}\right)$. Consequently $o(a)$ divides $s$; but since $o(a)=p_{k}^{\lambda}, s=p_{1}^{\mu_{1}} \cdots p_{k-1}^{\mu_{k-1}}$ and $p_{k} \neq p_{j}(j=1, \ldots, k-1)$, we conclude $o(a)=1$ and $a \in E(S)$. Thus we have proved that $\left(\prod_{i<k} P_{i}\right) \cap P_{k} \subseteq E(S)$; the opposite inclusion is clear, since $E(S) \subseteq P_{j}$ for every $j=1, \ldots, n$ by (1) and so $E(S) \subseteq \prod_{i<k} P_{i}$.

(3) Let $s \in S$, i.e. $s \in G_{\alpha}$ for some $\alpha \in Y$. Since $S$ is nilpotent, also $G_{\alpha}$ is nilpotent by Theorem 4.1 ; thus $G_{\alpha}$ is the direct product of its Sylow $p_{i}$-subgroups $\left(G_{\alpha}\right)_{p_{i}}$ and $s=g_{1} \cdots g_{t}$ with $g_{i} \in\left(G_{\alpha}\right)_{p_{i}} \subseteq P_{i}$. Consequently $s=q_{1} \cdots q_{n}$ with $q_{i} \in P_{i}(i=$ $1, \ldots, n)$, because those factors $q_{j}$ which do not occur in $s=g_{1} \cdots g_{t}$ can be added choosing the unity $e_{\alpha} \in G_{\alpha}$ for them ( $e_{\alpha} \in P_{i}$ for $i=1, \ldots, n$ trivially).

(4) Let $a \in P_{i} \cap P_{j}(i \neq j)$; then $o(a)=p_{i}^{r}=p_{j}^{t}$. Since $p_{i} \neq p_{j}$, it follows $r=t=0$ and thus $o(a)=1$, i.e. $a \in E(S)$ and $P_{i} \cap P_{j} \subseteq E(S)$. The opposite inclusion is trivial by Remark (2) above.

Applying Proposition 3.1(c) we conclude from Lemma 4.2 the following

COROLlaRY. If $S$ is a finite nilpotent Clifford semigroup, then every element admits a representation as a product of pairwise commuting elements in the Sylow subsemigroups of $S$, i.e. $s \in S$ implies that $s=q_{1} \cdots q_{n}$ with $q_{i} \in P_{i}$ and $q_{i} q_{j}=q_{j} q_{i}(i \neq j)$, $i, j=1, \ldots, n$.

Nilpotent groups $G$ are exactly those which are the direct product of their Sylow $p_{i}$-subgroups $(G)_{p_{i}}\left(p_{i}\right.$ a prime divisor of the order of $\left.G\right)$; thus every element of $G$ admits a unique factorisation into elements of $(G)_{p_{i}}(i=1, \ldots, k)$. For nilpotent Clifford semigroups $S$ we have by the corollary above indeed a representation of any element of $S$ as a product of commuting elements in the Sylow subsemigroups $P_{i}$ of $S$, but uniqueness is valid only "up to idempotent factors".

TheOREM 4.3. A finite Clifford semigroup $S$ is nilpotent iff every element $s \in S$ admits a representation as product of elements in the Sylow subsemigroups $P_{i}$ of $S$ unique up to idempotent factors, i.e. $s=a_{1} \cdots a_{n}=b_{1} \cdots b_{n}, a_{i}, b_{i} \in P_{i}(i=1, \ldots, n)$, $s \in G_{\alpha}$ imply $a_{i} e_{\alpha}=b_{i} e_{\alpha}(i=1, \ldots, n)$, where $e_{\alpha}$ is the identity of $G_{\alpha}$.

Proof. Necessity. Let $s=a_{1} a_{2} \cdots a_{n}$ with $s \in G_{\alpha}$ and $a_{i} \in G_{\alpha_{i}}$. Since $\alpha=\alpha_{1} \alpha_{2}$ $\cdots \alpha_{n}$ in $Y$, it follows that $\alpha \leqslant \alpha_{i}$. Thus $\alpha \alpha_{i}=\alpha$. However, $s=e_{\alpha} s=\left(e_{\alpha} a_{1}\right)\left(e_{\alpha} a_{2}\right)$ $\cdots\left(e_{\alpha} a_{n}\right)$ and $e_{\alpha} a_{i} \in G_{\alpha \alpha_{i}}=G_{\alpha}$; the uniqueness of $e_{\alpha} a_{1}, \ldots, e_{\alpha} a_{n}$ now follows since $G_{\alpha}$ is a nilpotent group (by Theorem 4.1) and since $a_{i} \in P_{i} \Rightarrow e_{\alpha} a_{i} \in P_{i}$.

Sufficiency. By Theorem 4.1 we need only to prove that every $G_{\alpha}, \alpha \in Y$, is nilpotent. By way of contradiction we suppose that there exists a group $G=G_{\alpha}$, $\alpha \in Y$, which has two different Sylow $p_{i}$-subgroups $G_{1}$ and $G_{2}\left(p_{i}\right.$ a prime divisor of the order of $G_{\alpha}$ ). Let $g \in G_{1} \subseteq G, g \notin G_{2}$; then with $e=e_{\alpha}$ we have a representation $g=e \cdots e g e \cdot \cdots e$ where $e \in P_{j}(j \neq i)$ and $g \in P_{i}$. Now every finite group is the product of its Sylow subgroups $G_{p_{j}}$; by Huppert [5, Hilfssatz 2.12(a)] $G_{p_{1}} \cap G_{p_{k}}$ $=\left\{e_{\alpha}\right\}\left(p_{j} \neq p_{k}\right)$ implies that $\left|G_{p_{1}} \cdots G_{p_{m}}\right|=\left|G_{p_{1}}\right| \cdots\left|G_{p_{m}}\right|=p_{1}^{r_{1}} \cdots p_{m}^{r_{m}}$, which is exactly the order of the group $G$. Thus $G=G_{p_{1}} \cdots G_{p_{m}}$; in particular $G=G_{p_{1}}$ $\cdots G_{2} \cdots G_{p_{m}}$, since $G_{2}$ is a Sylow $p_{i}$-subgroup of $G$. Consequently $g \in G$ can be 
written in the form $g=g_{1} \cdots g_{i} \cdots g_{m}$ with $g_{j} \in G_{p_{1}} \subseteq P_{j}\left(g_{i} \in G_{p_{i}}=G_{2}\right)$. By hypothesis these two representations of $g \in S$ are equal up to the idempotent factor $e_{\alpha}=e\left(g \in G_{\alpha}\right)$; thus $g_{1} e=e e=e, \ldots, g_{i} e=g e, \ldots, g_{m} e=e e=e$, i.e. $g_{1}=$ $e, \ldots, g_{i}=g, \ldots, g_{m}=e$, which implies $g=g_{i} \in G_{2}$, a contradiction.

The following two results are generalisations of well-known properties of finite nilpotent groups.

Proposition 4.4. A finite nilpotent Clifford semigroup $S(\neq E(S))$ has nontrivial center, i.e. $Z(S) \neq E(S)$.

Proof. Since $S \neq E(S)$, let $\beta=\min \left\{\alpha \in Y|| G_{\alpha} \mid>1\right\}$ in the finite semilattice $Y$. Since $G_{\beta}$ is nilpotent (by Theorem 4.1) and $\left|G_{\beta}\right|>1$, there exists $z \in Z\left(G_{\beta}\right)$ with $z \neq e_{\beta}$. To show that $z \in Z(S)$, let $a \in G_{\alpha} \subseteq S$. Then $a z, z a \in G_{\alpha \beta}$. If $\alpha \beta<\beta$, then $a z=z a=e_{\alpha \beta}$ by choice of $\beta$. On the other hand, if $\alpha \beta=\beta$, then the definition of product in $S$ and the fact that $z \in Z\left(G_{\beta}\right)$ imply that $a z=\varphi_{\alpha, \beta}(a) z=z \varphi_{\alpha, \beta}(a)=z a$. Thus $z \in Z(S)$.

REMARK. In the proof of the following proposition we shall make use of the following observation: if $N$ is any normal subsemigroup of a finite Clifford semigroup $S$, then the set $\left\{q_{j} \mid j=1, \ldots, m\right\}$ of prime divisors of the orders of the defining groups $H_{\beta}$ of $S / N$ is contained in the set $\left\{p_{i} \mid i=1, \ldots, n\right\}$ of prime divisors of the orders of the defining groups $G_{\alpha}$ of $S$. In fact: if $[s] \in S / N$ where $o(s)=k$, then $[s]^{k}=\left[s^{k}\right]=\left[e_{\alpha}\right]$ and $o([s])$ divides $k=o(s)$; consequently if $q_{j}$ is a prime divisor of $\left|H_{\beta}\right|$, then by the Sylow theorem there is an element $[a] \in H_{\beta}$ with $o([a])=q_{j}^{l_{j}}$; since $o([a])$ divides $o(a)=p_{1}^{k_{1}} \cdots p_{n}^{k_{n}}, q_{j}$ is one of the primes $p_{1}, \ldots, p_{n}$. Renumbering the $p_{i}(i=1, \ldots, n)$ in the evident ordering we can write $q_{1}=$ $p_{1}, \ldots, q_{m}=p_{m}$. The primes $p_{m+1}, \ldots, p_{n}$ missing in $\left\{q_{j}\right\}$ can be added, since for every idempotent $[e] \in S / N$ we have $o([e])=p_{j}^{0}$ with $j=m+1, \ldots, n$.

Proposition 4.5. If $S$ is a finite nilpotent Clifford semigroup, then for every normal subsemigroup $N$ of $S$ also $S / N$ is nilpotent.

Proof. By Lemma 2.4(a) $S / N$ is a Clifford semigroup again. With regard to the Remark above we have to show that the sets $Q_{i}=\left\{[a] \in S / N \mid o([a])=p_{i}^{r}, r \geqslant 0\right\}$, $i=1, \ldots, m$, are subsemigroups of $S / N$. For this end we shall show that for every $[s] \in Q_{i}$ there exists $a_{i} \in P_{i}$, such that $[s]=\left[a_{i}\right]$; for then $[a],[b] \in Q_{i}$ implies $[a b]=[a][b]=\left[a_{i}\right]\left[b_{i}\right]=\left[a_{i} b_{i}\right]$, thus

$$
[a b]^{p_{\prime}^{r}}=\left[a_{i} b_{i}\right]^{p_{\prime}^{r}}=\left[\left(a_{i} b_{i}\right)^{p_{\prime}^{\prime}}\right]=[e] \text { with }[e] \in E(S / N),
$$

since $a_{i}, b_{i} \in P_{i}$ implies by hypothesis that $a_{i} b_{i} \in P_{i}$; consequently $[a][b] \in Q_{i}$.

By the Corollary of Lemma 4.2, every $s \in S$ can be written in the form $s=c_{1}$ $\cdots c_{n}=c_{1} \cdots c_{i-1} c_{i+1} \cdots c_{n} c_{i}$ with $c_{j} \in P_{j}$. Let $s \in G_{\alpha}, c_{j} \in G_{\alpha}$; then $\alpha=\alpha_{1} \cdots \alpha_{n}$. If we show that $[s]=\left[c_{i} e_{\alpha}\right]$ for $[s] \in Q_{i}, e_{\alpha} \in G_{\alpha}$, then we can choose $a_{i}=c_{i} e_{\alpha} \in$ $P_{i} \cdot P_{i} \subseteq P_{i}$. This is equivalent to showing

$$
s s^{-1}=\left(c_{i} e_{\alpha}\right)\left(c_{i} e_{\alpha}\right)^{-1}, s\left(c_{i} e_{\alpha}\right)^{-1} \in N .
$$


We have $s s^{-1}=e_{\alpha}$ and $\left(c_{i} e_{\alpha}\right)\left(c_{i} e_{\alpha}\right)^{-1}=c_{i} c_{i}^{-1} e_{\alpha} \in G_{\alpha} \cdot G_{\alpha}=G_{\alpha}$ (since $\alpha \leqslant \alpha_{i}, i=$ $1, \ldots, n)$, consequently $\left(c_{i} e_{\alpha}\right)\left(c_{i} e_{\alpha}\right)^{-1}=e_{\alpha}$. Furthermore

$$
\begin{aligned}
s\left(c_{i} e_{\alpha}\right)^{-1} & =\left(c_{1} \cdots c_{i-1} c_{i+1} \cdots c_{n}\right)\left(c_{i} c_{i}^{-1}\right) e_{\alpha} \\
& =c_{1} \cdots c_{i-1} c_{i+1} \cdots c_{n} e_{\alpha_{i}} e_{\alpha}=c_{1} \cdots c_{i-1} c_{i+1} \cdots c_{n} e_{\alpha}
\end{aligned}
$$

(since $\alpha \leqslant \alpha_{i}$ ). Now $[s] \in Q_{i}$ implies that $o([s])=p_{i}^{r}$, thus $[s]^{p_{i}^{r}}=\left[s^{p_{i}^{r}}\right]=[e]$ for some $e \in E(S)$, i.e. $s^{p_{i}^{r}}$ belongs to $\operatorname{ker} \kappa(\varepsilon, N)=N$. If $p$ denotes $\max \left\{p_{i}^{r}, p_{i}^{r_{1}}\right\}$, where $p_{i}^{r_{i}}=o\left(c_{i}\right)$, then $s^{p}=c_{1}^{p} \cdots c_{i}^{p} \cdots c_{n}^{p}$ by the Corollary of Lemma 4.2, i.e. $s^{p}=c_{1}^{p}$ $\cdots e_{\alpha_{i}} \cdots c_{n}^{p}$. The system of linear congruences in $\mathbf{N}$ :

$$
p x \equiv 1\left(p_{1}^{r_{1}}\right), \ldots, p x \equiv 1\left(p_{i-1}^{r_{i-1}}\right), p x \equiv 1\left(p_{i+1}^{r_{i+1}}\right), \ldots, p x \equiv 1\left(p_{n}^{r_{n}}\right)
$$

has because of $\left(p, p_{j}^{r_{j}}\right)=1$ for all $j \neq i$ by the Chinese remainder theorem a solution $q \in \mathbf{N}$. Thus $\left(s^{p}\right)^{q}=c_{1}^{p q} \cdots e_{\alpha_{i}} \cdots c_{n}^{p q}=c_{1} \cdots e_{\alpha_{i}} \cdots c_{n}$; consequently

$$
\begin{aligned}
s^{p q} e_{\alpha} & =c_{1} \cdots e_{\alpha_{i}} \cdots c_{n} e_{\alpha}=c_{1} \cdots c_{i-1} c_{i+1} \cdots c_{n} e_{\alpha_{i}} e_{\alpha} \\
& =c_{1} \cdots c_{i-1} c_{i+1} \cdots\left(c_{i} c_{i}^{-1}\right) e_{\alpha}=s\left(c_{i} e_{\alpha}\right)^{-1} .
\end{aligned}
$$

Since the element $s^{p_{i}^{r}}$ belongs to $N$, also $s^{p q} e_{\alpha} \in N$ and we obtain $s\left(c_{i} e_{\alpha}\right)^{-1} \in N$.

For finite groups $G$ nilpotency is equivalent to the fact that the "ascending central series" of $G$ terminates in $G$ : if $Z_{0}=\{1\}$ and $Z_{i}$ denotes the complete inverse image of $Z\left(G / Z_{i-1}\right)$ under the natural homomorphism $G \rightarrow G / Z_{i-1}$, then there exists a $k \in \mathbf{N}$ such that $\{1\}=Z_{0} \subset Z_{1} \subset \cdots \subset Z_{k}=G$. For nilpotent Clifford semigroups the same is true defining the ascending central series in an analogous way.

THEOREM 4.6. If $S$ is a finite nilpotent Clifford semigroup $(\neq E(S))$, then the ascending central series of $S$ terminates in $S$, i.e. there is a $k \in \mathbf{N}$ such that $Z_{k}=S$, where $Z_{0}=E(S), Z_{1}=Z(S)$ and $Z_{i}$ is the complete inverse image of $Z\left(S / Z_{i-1}\right)$ under the natural homomorphism $S \rightarrow S / Z_{i-1}$.

Proof. First we have to show that $Z_{i}(i=0,1, \ldots)$ are normal subsemigroups of $S$ : for $i=0, Z_{0}=E(S)$ is normal, trivially. By way of induction suppose $Z_{i-1}$ is normal in $S$; then by Lemma $2.1 S / Z_{i-1}$ is defined and by Lemma 2.4(a) is a Clifford semigroup too; but $Z\left(S / Z_{i-1}\right)$ is normal in $S / Z_{i-1}$ by Lemma 2.1 again, so that by Lemma 2.3(a) the complete inverse image $Z_{i}$ of $Z\left(S / Z_{i-1}\right)$ under natural homomorphism $S \rightarrow S / Z_{i-1}$ is again normal in $S$.

Now since $S$ is nilpotent, $Z_{1}=Z(S) \neq E(S)$ by Proposition 4.4, i.e. $Z_{0}=E(S)$ $\subset Z_{1}=Z(S)$. Assume $Z_{0} \subset Z_{1} \subset \cdots \subset Z_{i}$ for some $i \in \mathbf{N}$. By Lemma 2.1 and what we have just proved $S / Z_{i}$ is defined and by Proposition 4.5 is nilpotent again. There are two possibilities for $S / Z_{i}$. First: $S / Z_{i}=E\left(S / Z_{i}\right)$; then by Lemma 2.2 we have $Z_{i}=S$ and the ascending central series terminates already with $k=i$. Second: $S / Z_{i} \neq E\left(S / Z_{i}\right)$; then by Proposition 4.4 we have $Z\left(S / Z_{i}\right) \neq E\left(S / Z_{i}\right)$. Thus the complete inverse image $Z_{i+1}$ of $Z\left(S / Z_{i}\right)$ under the natural homomorphism $\varphi$ : $S \rightarrow S / Z_{i}$ contains $Z_{i}$ properly, since for $Z_{i+1}=Z_{i}$ we had $\varphi\left(Z_{i+1}\right)=\varphi\left(Z_{i}\right)=$ $E\left(S / Z_{i}\right)$ by Lemma $2.3\left(\right.$ b), which would imply $Z\left(S / Z_{i}\right)=E\left(S / Z_{i}\right)$ by definition of 
$Z_{i+1}$, a contradiction. Consequently we get $Z_{0} \subset Z_{1} \subset \cdots \subset Z_{i} \subset Z_{i+1}$. But $S$ is finite, so that the first possibility has to occur for some $k \in \mathbf{N}$ and we obtain $Z_{k}=S$.

For (finite) groups $G$ the concept of "descending central series" is defined as follows: if $T, U$ are subsets of $G$ and if $[T, U]$ denotes the subgroup of $G$ generated by all the commutators $[t, u], t \in T, u \in U$, then for $K_{1}=G, K_{2}=\left[K_{1}, G\right]=[G, G]$, generally: $K_{i}=\left[K_{i-1}, G\right]$ we have $G=K_{1} \supset K_{2} \supset \cdots$. In case that $G$ is nilpotent, the descending central series terminates in the identity of $G$.

For inverse semigroups $S$ the sets $K_{i}(i=1,2, \ldots)$ can be defined similarly as those inverse subsemigroups of $S$ which are generated by all the commutators $[k, s]$, $k \in K_{i-1}, s \in S$, where $K_{1}=S$. We shall prove an analogous criterion for the nilpotency of finite Clifford semigroups using this concept. First we show that each $K_{i}$ is a normal subsemigroup of $S$ : for $i=1$ the assertion is trivial; for $i=2$ : $K_{2}=[S, S]=S^{\prime}$, the commutator subsemigroup of $S$, which is normal in $S$ (see $\S 3$ ); now let $K_{i-1}$ be normal in $S$, then by definition $K_{i}$ is an inverse subsemigroup of $S$ with $E(S) \subseteq K_{i}$ (since $e \in K_{i-1}$ and $e \in S$ imply $e=[e, e] \in K_{i}$ ); further $x \in S$, $a \in K_{i}$, i.e. $a=[r, s]$ with $r \in K_{i-1}, s \in S$, imply $x^{-1} a x=\left[x^{-1} r x, x^{-1} s x\right] \in K_{i}$ by the same argument as for $S^{\prime}$ in $\S 3$, since $x^{-1} r x \in K_{i-1}$ by hypothesis.

THEOREM 4.7. For a finite Clifford semigroup $S$ the following hold:

(1) If $Z_{k}=S$ for some $k \in \mathbf{N}$, then the descending central series of $S$ terminates in $E(S)$, i.e. $K_{j}=E(S)$ for some $j \in \mathbf{N}$, and $j \geqslant k+1$.

(2) If $K_{j}=E(S)$ for some $j \in \mathbf{N}$, then $S$ is nilpotent.

Proof. (1) First we show by induction that $K_{i} \subseteq Z_{k-i+1}(i=1,2, \ldots)$. For $i=1$ : $S=K_{1} \subseteq Z_{k}=S$. Suppose now that $K_{i-1} \subseteq Z_{k-i+2}$; then we get $K_{i}=\left[K_{i-1}, S\right] \subseteq$ $\left[Z_{k-i+2}, S\right]=T$. If $\varphi$ denotes the natural homomorphism $S \rightarrow S / Z_{k-i+1}$, we proceed to show that $\varphi(a) \in E\left(S / Z_{k-i+1}\right)$ for every $a \in T$ : if $a \in T$, then $a=[r, s]$ for some $r \in Z_{k-i+2}, s \in S$, or is a product of such commutators; consequently $\varphi(a)=[\varphi(r), \varphi(s)]$. Since $\varphi\left(Z_{k-i+2}\right)=Z\left(S / Z_{k-i+1}\right)$, we have $\varphi(r) \in$ $Z\left(S / Z_{k-i+1}\right)$, and further by definition of $\varphi: \varphi(s) \in S / Z_{k-i+1}$; thus $\varphi(r) \varphi(s)=$ $\varphi(s) \varphi(r)$; by Proposition 3.1(a) we get $\varphi(a)=[\varphi(r), \varphi(s)] \in E\left(S / Z_{k-i+1}\right)$, since $S / Z_{k-i+1}$ is a Clifford semigroup by Lemma 2.4(a). This means $\varphi(a)=\varphi(e)$ for some $e \in E(S)$ by the lemma of Lallement [6], i.e. $[a]_{\kappa}=[e]_{\kappa}$ for the congruence $\kappa=\kappa\left(\varepsilon, Z_{k-i+1}\right)$. By definition of $\kappa$ we conclude that $a^{-1} a=e$, ae $\in Z_{k-i+1}$, so that by the definition of a congruence pair follows $a \in Z_{k-i+1}$. Consequently we obtain $T \subseteq Z_{k-i+1}$ and $K_{i} \subseteq T \subseteq Z_{k-i+1}(i=1,2, \ldots)$. For $i=k+1$ this gives $K_{k+1} \subseteq$ $Z_{0}=E(S)$; conversely $K_{k+1}$ is normal in $S$, so that $E(S) \subseteq K_{k+1}$ and equality follows: $K_{k+1}=E(S)$. Generally, since $E(S)=K_{k+1} \subseteq K_{k} \subseteq \ldots$, we have $K_{j}=$ $E(S)$ for all $j \geqslant k+1$.

(2) Conversely let $K_{j}=E(S)$ for some $j \in \mathbf{N}$; then it is easily seen that $K_{j}\left(G_{\alpha}\right) \subseteq$ $K_{,}(S)=E(S)$ for each of the defining groups $G_{\alpha}, \alpha \in Y$, of $S$. Since $K_{j}\left(G_{\alpha}\right) \subseteq G_{\alpha}$ for every $\alpha \in Y$ and since $G_{\alpha}$ has exactly one idempotent, the identity $e_{\alpha}$, we get $K_{j}\left(G_{\alpha}\right)=\left\{e_{\alpha}\right\}$. This means that for every $\alpha \in Y$ there is $j_{\alpha} \leqslant j$ such that $K_{j}\left(G_{\alpha}\right)=$ $\left\{e_{\alpha}\right\}$; thus by Huppert [5, Satz III.2.3], every $G_{\alpha}$ is nilpotent, and consequently $S$ is nilpotent, too (by Theorem 4.1). 
Applying Theorem 4.6 we obtain the following characterisation.

COROLlaRY. A finite Clifford semigroup $S$ is nilpotent iff its descending central series terminates in $E(S)$, or equivalently: iff its ascending central series terminates in $S$.

Another necessary and sufficient condition for the nilpotency of a Clifford semigroup is the following:

THEOREM 4.8. A finite Clifford semigroup $S$ is nilpotent iff every homomorphic image $T$ of $S$ with $T \neq E(T)$ has nontrivial center (i.e. $Z(T) \neq E(T)$ ).

Proof. Sufficiency. For $T=S$ we have by hypothesis $Z(S) \neq E(S)$. By Lemma $2.1(\varepsilon, Z(S))$ is a congruence pair, thus $S / \kappa(\varepsilon, Z(S))$ is a homomorphic image of $S$. If $S / Z(S)=E(S / Z(S))$, then $Z(S)=S$ by Lemma 2.2 and the ascending central series is $Z_{0}=E(S) \subset S=Z_{1}$; thus $S$ is nilpotent by the Corollary of Theorem 4.7. If $S / Z(S) \neq E(S / Z(S))$, then $Z(S / Z(S))$ is nontrivial by hypothesis; thus we obtain for the complete inverse image $Z_{2}$ of $Z(S / Z(S))$ under the natural homomorphism $S \rightarrow S / Z(S)=S / Z_{1}$ that $Z_{1} \subset Z_{2}$ (see the proof of Theorem 4.6). This inclusion being strict we get after a finite number of such steps: $Z_{0} \subset Z_{1} \subset \cdots \subset$ $Z_{k}=S$ (since $S$ is finite) and $S$ is nilpotent by the Corollary of Theorem 4.7.

Necessity. We show, that every homomorphic image $T$ of the nilpotent Clifford semigroup $S$ is nilpotent (this implies by Theorem 4.4, that $Z(T) \neq E(T)$ ). First we note that $K_{j}(S)=E(S)$ for some $j \in \mathbf{N}$ (the Corollary of Theorem 4.7). If $\varphi: S \rightarrow T$ denotes the defining homomorphism, then we have $\varphi\left(K_{j}(S)\right)=\varphi(E(S))=E(T)=$ $K_{j}(\varphi(S))$ by Lallement [6]. Generally: $\varphi\left(K_{i}(S)\right)=K_{i}(\varphi(S)), i=1,2, \ldots$ In fact: for $i=1$ we have $K_{1}(S)=S$ and $\varphi\left(K_{1}(S)\right)=\varphi(S)=T=K_{1}(T)=K_{1}(\varphi(S))$. Assume that $\varphi\left(K_{i-1}(S)\right)=K_{i-1}(\varphi(S))$ has already been shown: then $\varphi\left(K_{i}(S)\right)=$ $\varphi\left(\left[K_{i-1}(S), S\right]\right)=\left[\varphi\left(K_{i-1}(S)\right), \varphi(S)\right]=\left[K_{i-1}(\varphi(S)), \varphi(S)\right]=K_{i}(\varphi(S))$. For $i=j$ we get: $E(T)=\varphi(E(S))=\varphi\left(K_{j}(S)\right)=K_{j}(\varphi(S))=K_{j}(T)$, so that by the Corollary of Theorem 4.7 also $T$ is nilpotent.

The proof of the necessity above generalises Proposition 4.5 to

COROLlARY. Every homomorphic image of a finite nilpotent Clifford semigroup is again nilpotent.

As a last criterion on nilpotency of Clifford semigroups we show

THEOREM 4.9. A finite Clifford semigroup $S$ is nilpotent iff elements of $S$ having relatively prime orders commute.

Proof. If all elements of relatively prime order in $S$ commute, so do in particular all $a, b \in G_{\alpha}(\alpha \in Y)$, if their order is relatively prime; thus every group $G_{\alpha}$ is nilpotent (by Huppert [5, III.2.3]) and so by Theorem 4.1 $S$ is nilpotent, too.

Conversely let $S$ be nilpotent, $a, b \in S$ elements with relatively prime orders; then $a \in G_{\alpha}, b \in G_{\beta}$ and $a b, b a \in G_{\alpha \beta}=G_{\gamma}(\alpha, \beta, \gamma=\alpha \beta \in Y)$, where by Theorem 4.1 $G_{\gamma}$ is nilpotent. We have $a b=\varphi_{\alpha, \gamma}(a) \varphi_{\beta, \gamma}(b) \in G_{\gamma}$; since the order of the image of $a$ resp. $b$ under the corresponding structure homomorphism $\varphi_{\alpha, \gamma}$ resp. $\varphi_{\beta, \gamma}$ is a divisor of the order of $a$ resp. $b$, the orders of $\varphi_{\alpha, \gamma}(a)$ and $\varphi_{\beta, \gamma}(b)$ in $G_{\gamma}$ are relatively prime 
also; consequently they commute (Huppert [5, III.2.3]) and we finally have $a b=$ $\varphi_{\alpha, \gamma}(a) \varphi_{\beta, \gamma}(b)=\varphi_{\beta, \gamma}(b) \varphi_{\alpha, \gamma}(a)=b a$.

Summarising the results of Theorems 4.3, 4.6, 4.7, 4.8 and 4.9 we get

MAIN THEOREM. If $S$ is a finite inverse semigroup with central idempotents, then the following properties of $S$ are equivalent:

(a) $S$ is nilpotent.

(b) $S$ is the product of its Sylow subsemigroups, representations being unique up to idempotent factors.

(c) The ascending central series of $S$ terminates in $S$.

(d) The descending central series of $S$ terminates in $E(S)$.

(e) Every nontrivial homomorphic image of $S$ has a nontrivial center.

(f) Elements of $S$ having relatively prime orders commute.

Finally we prove two results on minimal normal subsemigroups resp. homomorphic images of nilpotent Clifford semigroups.

Proposition 4.10. If $N$ is a minimal normal subsemigroup of a nilpotent Clifford semigroup $S$, then $N \subseteq Z(S)$; in particular $(N, \cdot)$ is commutative.

Proof. It can be easily seen that $[N, S]$ is a normal subsemigroup of $S$. We show that $[N, S] \subset N$ : clearly $[N, S] \subseteq N$, since $[n, s]=n^{-1}\left(s^{-1} n s\right) \in N$ for every $n \in N$, $s \in S$; assume by way of contradiction, that $[N, S]=N$; then $[N, S] \subseteq[S, S]$ and [[ $N, S], S] \subseteq[[S, S], S]$, i.e. $[N, S] \subseteq K_{3}$, and so on, until: $N=[N, S] \subseteq K_{j}=E(S)$ for some $j \in \mathbf{N}$, since $S$ is nilpotent (the Corollary of Theorem 4.7); but $N$ is normal, thus $E(S) \subseteq N$ and we get $N=E(S)$, a contradiction to the minimality of $N$. Now $[N, S] \subset N$ and the minimality of $N$ again imply $[N, S]=E(S)$; this means $[n, s] \in E(S)$ for all $n \in N, s \in S$, so that $n s=s n$ for all $n \in N, s \in S$ by Proposition 3.1.

Proposition 4.11. Let $S$ be a Clifford semigroup, $\varphi(S)=T$ a homomorphic image of $S, \kappa=\kappa(\xi, N)$ the corresponding congruence. Then $T$ is nilpotent iff $K_{i}(S) \subseteq N$ for some $i \in \mathbf{N}$.

Proof. If $\varphi(S)=T$ is nilpotent, then by the Corollary of Theorem 4.7 there exists some $i \in \mathbf{N}$ such that $K_{i}(T)=E(T)$, i.e. $K_{i}(\varphi(S))=E(\varphi(S))$. By Theorem 4.8, proof of necessity, we conclude $\varphi\left(K_{i}(S)\right)=K_{i}(\varphi(S))=E(\varphi(S))$. This implies that for every $a \in K_{i}(S)$ we have $\varphi(a)=[a]_{\kappa}=[e]_{\kappa}$ for some $e \in E(S)$ (by Lallement [6]); consequently $a \kappa(\xi, N) e$ and $a \in \operatorname{ker} \kappa=N$, i.e. $K_{i}(S) \subseteq N$ for this $i \in \mathbf{N}$.

Conversely let $K_{i}(S) \subseteq N$ for some $i \in \mathbf{N}$. We have already proved (before Theorem 4.7), that $K_{i}(S)$ is a normal subsemigroup of $S$; thus by Mitsch [7, Proposition 2], $\left(\varepsilon, K_{i}(S)\right)$ is a congruence pair of $S$. Now in the natural ordering of congruence pairs given by Petrich [8, Corollary 4.5], $\left(\varepsilon, K_{i}(S)\right) \leqslant(\xi, N)$, thus $\rho=\rho\left(\varepsilon, K_{i}(S)\right) \leqslant \kappa=\kappa(\xi, N)$. Consequently $(S / \rho) /(\kappa / \rho) \cong S / \kappa$ by the second isomorphism theorem (see Howie [3, Theorem I.5.6]). Further $S / \rho$ is nilpotent, since by Lemma 2.3 (b) we have $\varphi\left(K_{i}(S)\right)=E(S / \rho)\left(K_{i}(S)=\operatorname{ker} \rho\right)$, thus by the proof of the necessity in Theorem 4.8 follows $K_{i}(\varphi(S))=E(\varphi(S))$ and consequently 
$\varphi(S)=S / \rho$ is nilpotent by Theorem 4.7(2). Since every homomorphic image of a nilpotent Clifford semigroup is again nilpotent (the Corollary of Theorem 4.6), also $(S / \rho) /(\kappa / \rho)$ is nilpotent and so is $S / \kappa=\varphi(S)=T$.

ADDED IN PROOF. Using the commutator calculus in inverse semigroups developed in $\S 3$ "solvable" Clifford semigroups can be defined; these will be considered in a subsequent paper.

\section{REFERENCES}

1. A. H. Clifford, Semigroups admitting relative inverses, Ann. of Math. (2) 42 (1949), 1037-1049.

2. A. H. Clifford and G. B. Preston, The algebraic theory of semigroups. Vol. I, Amer. Math. Soc., Providence, R.I., 1967.

3. J. M. Howie, An introduction to semigroup theory, Academic Press, New York, 1976.

4. The maximum idempotent-separating congruence on an inverse semigroup, Proc. Edinburgh Math. Soc. (2) 14 (1964/65), 71-79.

5. B. Huppert, Endliche Gruppen. I, Springer-Verlag, Berlin and New York, 1967.

6. G. Lallement, Congruences et équivalences de Green sur un demigroup régulier, C. R. Acad. Sci. Paris Ser. A 262 (1966), 613-616.

7. H. Mitsch, $N$-simple inverse semigroups, Math. Japon. 26 (1981), 349-358.

8. M. Petrich, Congruences on inverse semigroups, J. Algebra 55 (1978), 231-256.

Institut fUr Mathematik, Strudlhofgasse 4, A-1090 Wien, Austria 